\title{
Serum levels of the IL-6 family of cytokines predict prognosis in renal cell carcinoma (RCC)
}

\author{
Gigja Gudbrandsdottir ${ }^{1,4}$ (D) Helene H. Aarstad ${ }^{5} \cdot$ Leif Bostad $^{2,4} \cdot$ Karin M. Hjelle $^{1,4} \cdot$ Hans J. Aarstad $^{3,4}$. \\ $\varnothing_{\text {ystein Bruserud }}{ }^{5,6} \cdot$ Tor Henrik Anderson Tvedt $^{5,6} \cdot$ Christian Beisland $^{1,4}$
}

Received: 22 April 2020 / Accepted: 25 June 2020 / Published online: 3 July 2020

(c) The Author(s) 2020

\begin{abstract}
Purpose An improved understanding of RCC immunology should shed further light on RCC tumor biology. Our objective was to study to what extent serum levels of the IL-6 family of cytokines at diagnosis were relevant to survival.

Methods A total of 118 consecutively patients with RCC, in which the tumor was surgically removed at Haukeland University Hospital during the period from 2007 to 2010, were included. The patients were followed-up for 10 years. The morning before surgery blood was sampled and serum frozen, with levels of IL-6, IL-27, IL-31, OSM, CNTF, IL-6R $\alpha$ and gp130 determined. Results Among patients with the highest quartile of IL-6 $(>8 \mathrm{pg} / \mathrm{ml})(n=29)$, six of nine who had metastasis at diagnosis had such high IL-6 values. Among presumed radically treated patients, a high IL-6 and IL-27 strongly predicted recurrence. In particular, the predictions among patients with large (diameter $>7 \mathrm{~cm}$ ) tumors were excellent regarding both IL-6 and IL-27 values. High gp130 serum levels predicted an overall survival (OS) among RCC patients with large tumors. Patients with a high IL-6 exhibited a strong expression of IL-6 in endothelial- and vascular smooth muscle cells. Moreover, the level of intra-tumoral CD3-positive cells predicted survival.

Conclusions IL-6 and IL-27 seem to play a role in RCC biology. IL-6 enables the pinpointing of metastatic condition at diagnosis, as well as together with IL-27, the predicting of survival and recurrence. Endothelial cells and vascular smooth muscle cells are both suggested as important sources of IL-6.
\end{abstract}

Keywords IL-6 · IL-27 · gp130 · Survival $\cdot$ Recurrence $\cdot$ Renal cell carcinoma

Electronic supplementary material The online version of this article (https://doi.org/10.1007/s00262-020-02655-z) contains supplementary material, which is available to authorized users.

Gigja Gudbrandsdottir

gigjagud@gmail.com

1 Surgical Clinic, Department of Urology, Haukeland University Hospital, 5021 Bergen, Norway

2 Department of Pathology, Haukeland University Hospital, Bergen, Norway

3 Department of Otolaryngology/Head and Neck Surgery, 5021 Bergen, Norway

4 Department of Clinical Medicine, University of Bergen, 5021 Bergen, Norway

5 Department of Clinical Science, University of Bergen, 5021 Bergen, Norway

6 Department of Internal Medicine, Haukeland University Hospital, Bergen, Norway

\section{Introduction}

Cancer diseases are major global killers of humans [1]; thus, there is an urgent need to better understand these diseases. It is generally accepted that carcinomas are caused by somatic DNA mutations with a consequent dysregulation of the affected cells. Furthermore, it has been known that carcinomas are not only built by actual carcinoma cells, but also, e.g., by intra-tumor immune cells. Biological information from carcinomas is collected with a biopsy, or from a resected tumor, both of which are instant pictures of a long-term ongoing process. One important source of tumor biology knowledge is serum samples, of which tumorassociated secretory interleukins/cytokines contribute, with the study of this cytokine reservoir in cancer patients being the primary goal of this study. One primary validity criteria of all cancer studies is the association to prognosis, so we will therefore presently use survival as our readout variable. 
Renal cell cancer (RCC) is the ninth most common cancer in men and 14th most common cancer in women. In 2018, 175,098 deaths by RCC were estimated, making it the 14th most common cause of global cancer deaths [2]. $\mathrm{RCC}$ represents one of the major immunogenic carcinomas [3]. Over the last few years, biological therapy has gained importance as a treatment for metastatic RCC, mostly by VEGF blockage [4]. Recently, modern immune therapy has also been introduced [5].

IL-6 is a cytokine produced by, e.g., macrophages, Th2 cells, B cells, astrocytes, endothelial cells, adipocytes and some tumor cells [6]. IL-6 has been shown to promote tumor proliferation, metastases and symptoms of cachexia [6]. In a review paper, the IL-6 serum level at diagnosis was significantly correlated to survival in $82 / 101$ series, comprising 9917 out of 11,583 patients with 23 different cancer types [7].

IL-6 regulates inflammation by two main pathways: The classic signaling, in which it binds to a membrane-bound IL- $6 \alpha$ receptor expressed in only a few cell types and then secondarily to membrane-bound gp130 (signal transducing receptor glycoprotein $130 \mathrm{kDa}$ ) present in many cells [8]. The trans signaling IL- 6 binds to membrane gp130 through a primary binding to serum IL-6R $\alpha$ [9]. The classical signaling stimulates the regenerative and anti-inflammatory activity, whereas trans signaling has more general stimulatory effects [9]. When IL-6/IL-6R $\alpha$ binds to gp130, three signaling pathways may be activated: JAK-STAT, RasERK cascade or P13K-Akt signaling. Through all three ways of trans signaling, IL-6 promotes the growth of cancer cells, whereas STAT3 IL-6 also promotes tumor cells' ability to escape apoptosis [10]. On the other hand, soluble gp130 receptor serves as a decoy receptor that inhibits the function of IL-6/IL-6R $\alpha$ complex [11].

Several other cytokines also share the use of gp130 subunit receptor. These cytokines are collectively named the IL-6 family of cytokines [8, 12], which has several members, including IL-11, IL-27, IL-31, ciliary neurotrophic factor (CNTF), leukemia inhibitory factor (LIF), oncostatin M (OSM) and cardiotrophin-like cytokine factor 1 (CLC) [13]. The receptor signaling complexes for IL- 6 and IL-11 contain a gp130 homodimer, whereas other family members signal via a heterodimeric receptor complex containing gp130 [13].

IL-6 has been shown to be secreted from RCC cells exposed to hypoxia, and hypothesized to contribute to RCC invasion and the development of metastasis [14-16]. In RCC serum IL-6 levels have been associated with extended tumor stage, grade and metastatic progression [16].

Regarding other IL-6 family cytokines, Pu et al. [17] showed that two polymorphisms in the IL-27 gene were associated with an increased risk for RCC. IL-27 acts through a receptor consisting of IL-27R $\alpha$ and gp130, which mediates signaling mostly through STAT1 and STAT3, though similarly to IL-6. IL 27-R $\alpha$ is present on B, T and NK cells, neutrophils, monocytes and mast cells, as well as in lower levels in macrophages, hepatocytes, keratinocytes and endothelial cells [18]. IL-27 has demonstrated antitumor activity in prostate cancer, multiple myeloma, non-small cell lung cancer and ovarian cancer cell lines [18]. In contrast, high serum levels of IL-27 in breast and gastroesophageal cancer are correlated with advanced stage [18].

Tumor diameter, measured by CT prior to surgery, is a strong indicator for survival [19]. Most RCC-caused deaths occur in patients with tumors $>7 \mathrm{~cm}$ in diameter. Hence, we have aimed in particular at studying large RCC tumors as to evidence for cytokine involvement.

It is also of interest to study tumor tissue, both for the source of secretion and as a potential target [20]. We have therefore studied the level of macrophages and $\mathrm{T}$ lymphocytes in and around tumors, as well as IL-6 and IL-6 receptor levels on endothelial cells, macrophages and $\mathrm{T}$ lymphocytes, both in and adjacent to the tumors in patients with a high serum IL-6.

In this study, we have aimed at investigating whether the IL-6 family of cytokine members and pertinent cytokines receptors levels, both in serum preoperatively and in tumor tissue, relate to RCC biology by studying the prognostic value of these cytokine/receptor levels at diagnosis.

\section{Material and methods}

\section{Patients}

From the kidney cancer database at Haukeland University Hospital, we identified 159 consecutive patients treated with nephron sparing surgery (NSS), a radical nephrectomy (RN) or a cyto-reductive nephrectomy at our institution between January 1, 2007 and March 31, 2010. All histological subtypes and stages were included. For IL-6 analyses, 118 patients with appropriate blood samples were available, while for the other cytokine analyses 97 patients were available. Attrition analyses revealed no difference in regard to descriptive statistics between individuals registered in the database who had blood samples bio-banked and those who did not. Most patients were male $[n=88(75 \%)]$, the mean age was 63 years (median 64, IQR 55-73) and the mean tumor size was $6.3 \mathrm{~cm}$ (median 5.3, interquartile range IQR $3.7-8.7)$. A radical nephrectomy was performed in $66 \%$ $(n=75)$ of the patients.

All patients were followed-up to January 18 , $2018 / 10$ years or time of death, and the information registered. The follow-up flow chart at Haukeland University Hospital, which is based on Leibovich score (stage, lymph nodes, tumor size, nuclear grade and tumor necrosis) has been previously reported [21]. The mean observation time 
was 99 (median 105, IQR 95-120) months. During the observation period, 20 patients died from RCC, while 19 patients died from other causes. A total of 14 patients (12\%), presumed radically treated, developed metastases during the follow-up period. Our institutional Follow-up regime has been described in detail by our group [22]. The Regional Committee for Medical Research Ethics in Western Norway (78/05), the study and the Norwegian Social Science Data Services all approved the database. All patients signed informed consent forms at inclusion.

\section{Tumor assessment}

Patients were staged according to the 2009 TNM classification system, and the tumor histology was graded according to the Fuhrman nuclear criteria [23].

\section{Laboratory cytokine assessment}

Preoperative blood samples were drawn on the morning of the surgery, and serum was frozen at $-80 / 150{ }^{\circ} \mathrm{C}$ until analysis. Serum IL-6 was detected using the Luminex immune-bead technology and a high-sensitivity kit (Invitrogen/Biosource, Carlsbad, CA, USA). In short, antibody-coupled beads were incubated with serum and incubated with a biotinylated detection antibody, before finally being incubated with streptavidin-phycoerythrin. Samples were then read by the Luminex's laser-based fluorescent analytical test instrument Luminex ${ }^{\circledR} 100^{\mathrm{TM}}$ (Luminex Corporation Austin, TX, USA). Gp130, IL-27, IL-31, IL-6R $\alpha$, OSM, and CNTF measured with the same method: Human Premixed Multi-Analyte Kit from R\&D system, and the latter by the use of the Milliplex map kit Human Pituitary Magnetic Bead Panel 1 (Millipore, SigmaAldrich, Oslo, Norway).

\section{Immunohistological assessment}

Tumor tissue from patients with the highest IL-6 serum levels $(n=29)$ was investigated, with one representative block selected from each case. The selected slide contained both tumor tissue corresponding to the tumor ISUP grade and an area bordering on and comprising kidney parenchyma (interphase zone). An experienced uropathologist classified all the RCCs based on hematoxylin and eosin-stained sections.

Immunohistochemistry was performed using the automated benchmark ultra-system (Ventana-Diagnostics Roche). Four-micrometer sections from the formalinfixed paraffin embedded (FFPE) tissue blocks were de-paraffinized and rehydrated, while antigen retrieval was done by conditioning the cells in a TRIS-based buffer (CC1, Ventana) and heating. After endogenous peroxidase blocking, the slides were incubated with the primary antibodies. Detection was performed by OptiView ${ }^{\circledR}(\mathrm{OV})$ and UltraView ${ }^{\circledR}$ (UV) DAB detection kits (Ventana Medical Systems), with hematoxylin used as a counterstain. Human spleen and lymph node sections were used as positive controls, while for negative controls, primary antibodies were omitted (Supplementary Table 1).

The whole tumor area in the slide was examined and the subjective impression of density and number of positive cells were scored semi-quantitatively and subjectively. The proportion of IL-6 and IL6R-positive tumor cells were scored as "no positive tumor cells" (0), "less than $10 \%$ positive tumor cells" $(1+)$, "10-50\% positive tumor cells" $(2+)$, or "more than $50 \%$ positive tumor cells" $(3+)$. For CD3, CD68 and FOXP3, 1+ means slight and scattered infiltration, $2+$ moderate infiltration and $3+$ the dense infiltration of positive cells in more than $50 \%$ of the area.

\section{Statistical analysis}

Comparisons between groups were performed with crosstables and exact Chi-square test, Mann-Whitney $U$ test and $T$ test for categorical, ordinal and continuous data, respectively. A patient's serum levels of IL- $6 \geq 8 \mathrm{pg} / \mathrm{ml}$ (the uppermost quartile), and of IL-27 for the uppermost quartile, were defined as high. The multiple logistic regression models were performed in a backward likelihood ratio (LR) test manner without a pre-selection of the variables.

Kaplan-Meier analyses were used to estimate DSS and recurrence-free survival (RFS). For a survival comparison between different groups, a log rank test was used. A Cox proportional hazard model was used to determine DSS and RFS predictions after adjusting for other variables affecting survival in univariate analyses. Correlations between variables were calculated using Kendall analyses, while ROC curves were used to calculate predictive value, sensitivity and specificity of IL-6/IL-27 as to recurrence. For all statistical analyses, a $p$ value of less than 0.05 was considered statistically significant, and calculations were performed using the IBM $^{\circledR}$ SPSS $^{\circledR}$ Statistics software (Release 24.0).

\section{Results}

\section{IL-6 family cytokines versus tumor characteristics in patients presumed radically treated}

The patients $(n=109)$ were divided into two groups, those with a low (IL-6 $<8 \mathrm{pg} / \mathrm{ml}$ ) vs. high (IL-6 $\geq 8 \mathrm{pg}$ / $\mathrm{ml})$ IL-6 values at diagnosis. The groups did not differ in RCC subtype, tumor size, pathological stage, nuclear grade or other known predictive factors. Histological positive 
Fig. 1 a The panels show low (left) and high (right) scores with tumor area CD3 staining lymphocytes. b The panels show renal tissue outside the tumor: to the left small arteries showing IL-6 positivity, and to the right interlobular artery showing a strong IL-6 expression in medial smooth muscle cells
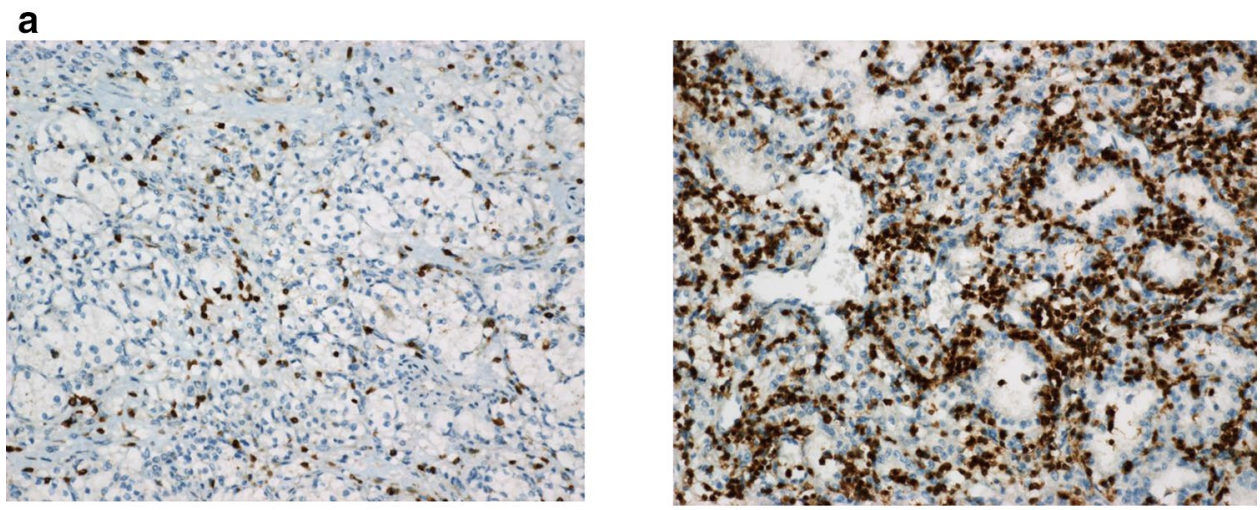

b

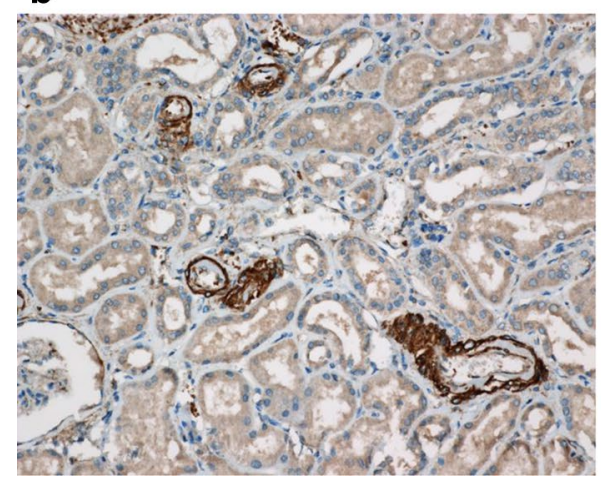

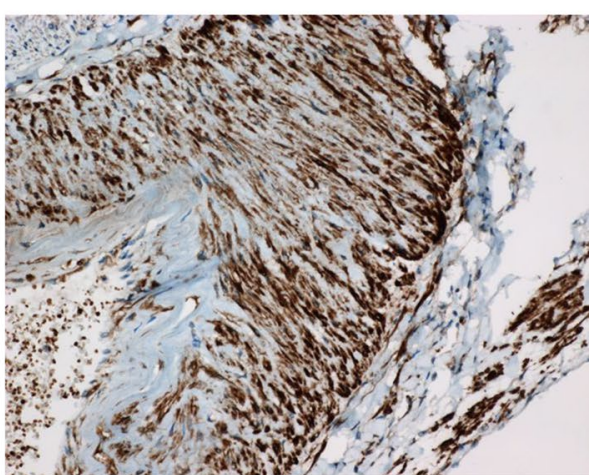

margins $(p=0.05)$ and pT stage $(p=0.054)$ differences were borderline differentiating between the patient groups (Supplementary Table 2). Immunohistochemistry was done in patients with high Il-6 in serum (Fig. 1).

\section{IL-6 family cytokines and soluble receptors recurrence prediction}

IL-6 levels predicted recurrence, both by Kaplan-Meier survival analysis $(p=0.001)$ (Fig. 2a) and utilizing a Cox multivariate regression analysis, with age, gender and tumor size additionally included as covariates (HR 7.13, CI 2.23-22.8; $p=0.001$ ) (Table 1). IL-27 showed a significant prediction of recurrence, analyzed by Kaplan-Meier analysis ( $p=0.026)$ (Fig. 2b) and multivariate Cox regression analysis, with covariates being age, gender and tumor size (HR 6.89; CI 1.56-30.4; $p=0.011$ ) (Table 1).

If both IL-6 and IL-27 were included to one Cox multivariate regression analysis, the recurrence of those presumably cured was predicted by IL-6 $(p=0.004)$, but not regarding IL-27 $(p=0.082)$ (Table 2).

If the patients were grouped by tumor size $( \pm 7 \mathrm{~cm})$ and studied by Kaplan-Meier analyses, both IL-6 $(p=0.014)$ (Fig. 2c) and IL-27 ( $p=0.001)$ (Fig. 2d) predicted recurrence among patients with large tumors (diameter $>7.0 \mathrm{~cm}$ ). Regarding gp130 levels there was not a statistic significance $(p=0.082)$ (Fig. 2e).

\section{IL-6 family cytokines and soluble receptors vs. DSS}

IL-6 predicted DSS in both Kaplan-Meier analysis $(p<0.001)$ (Fig. 3a) and multivariate regression analysis, including gender, age and tumor size (HR 4.82; CI $1.96-11.86 ; p=0.001$ ) (Table 1). In regard to IL-27, there was a borderline DSS prediction in the Kaplan-Meier analysis $(p=0.052)$ (Fig. 3b).

If both IL-6 and IL-27 were included in one Cox multivariate regression analysis for DSS, only IL-6 levels were predicted (HR 20.7; CI 2.6-44.4; $p=0.001$ ) (Table 2).

IL-6, gp130 and IL-6R $\alpha$ were included to one DSS multivariate analysis that also included gender, age and tumor size in one Cox regression survival model. Subsequently, IL-6 $(p<0.001)$ and IL-6R $\alpha(p=0.02)$, but not gp 130, showed survival prediction (Table 2).

If analyzed by tumor size, patients with a tumor diameter from a 4 to $7 \mathrm{~cm} \mathrm{IL-6} \mathrm{level} \mathrm{predicted} \mathrm{survival}$ by Kaplan-Meier analysis $(p=0.001)$ (Fig. 3c). The same was the case with large tumors (tumor diameter $>7.0 \mathrm{~cm}$ ) $(p=0.02)$ (Fig. 3g). When IL-27 levels were analyzed by size, it was determined that a survival prediction was found among the patients with large tumors (diameter $>7 \mathrm{~cm}$ ) $(p=0.025$ ) (Fig. 3d). Including only tumors $>7 \mathrm{~cm}$, s-gp130 levels exhibited no survival prediction $(p=0.09)$ 

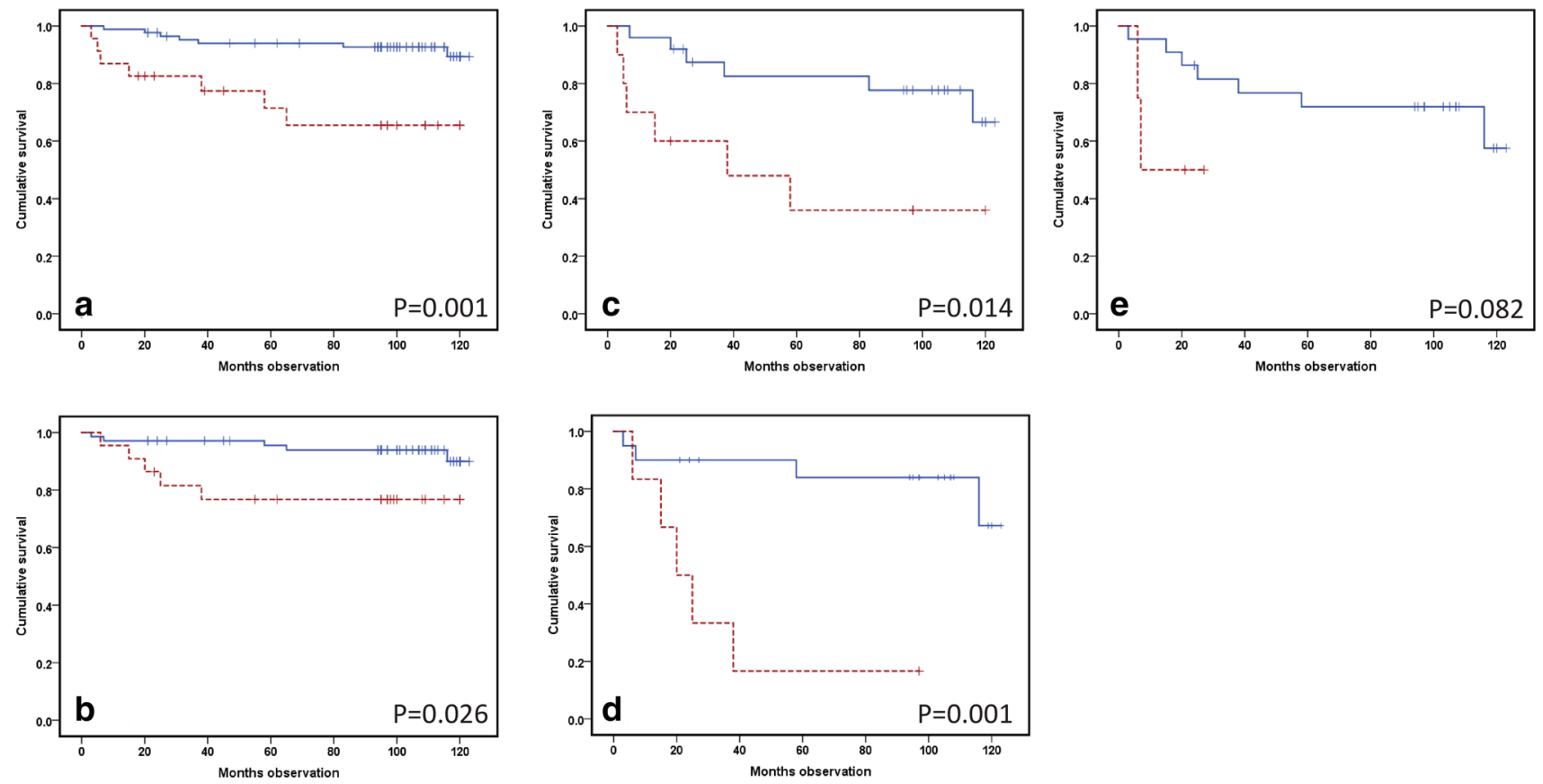

Fig. 2 Kaplan-Meier recurrence curves from IL-6 and some family members, as analyzed by Luminex in the serum of assumed radically treated renal cell carcinoma (RCC) patients, sampled prior to surgery. The blue line represents a low value, whereas the red dotted line indicates a high value. Differences between the groups are examined in log-rank tests and presented with $p$ values. a IL-6 recurrence prediction among 109 RCC patients (low $(<8 \mathrm{pg} / \mathrm{ml})$ : $n=86$ and high $(\geq 8 \mathrm{pg} / \mathrm{ml}): n=23)$. b IL-27 prediction of recurrence in $91 \mathrm{RCC}$ patients (low: $n=69$ and high: $n=22$ ). c-e Recurrence prediction of IL-6, IL-27, and gp130 in patients with large $(>7 \mathrm{~cm})$ RCC tumors (low: three lower quartiles)/high: highest quartile). c IL-6: $n=35$ (25/10). d IL-27: $n=26$ (20/6). e gp130: $n=26(22 / 4)$
Table 1 Recurrence and survival predictions from IL-6 and IL-27 in Cox regression analyses

\begin{tabular}{|c|c|c|c|c|c|c|c|c|}
\hline & \multicolumn{4}{|c|}{ Univariate } & \multicolumn{4}{|c|}{$\begin{array}{l}\text { Multivariate including age, gender and } \\
\text { tumor size }\end{array}$} \\
\hline & \multirow[t]{2}{*}{ HR } & \multicolumn{2}{|c|}{$95 \% \mathrm{CI}$} & \multirow[t]{2}{*}{$p$ value } & \multirow[t]{2}{*}{ HR } & \multicolumn{2}{|c|}{$95 \% \mathrm{CI}$} & \multirow[t]{2}{*}{$p$ value } \\
\hline & & Lower & Upper & & & Lower & Upper & \\
\hline \multicolumn{9}{|c|}{ Recurrence in presumed cured patients } \\
\hline IL-6, $n=109$ & 4.99 & 1.74 & 14.3 & 0.003 & 7.13 & 2.23 & 22.8 & 0.001 \\
\hline IL-27, $n=91$ & 3.77 & 1.08 & 13.2 & 0.038 & 6.89 & 1.56 & 30.4 & 0.011 \\
\hline \multicolumn{9}{|c|}{ Disease-specific survival in all included patients } \\
\hline IL-6, $n=118$ & 4.97 & 2.06 & 12.0 & $<0.001$ & 4.82 & 1.96 & 11.9 & 0.001 \\
\hline IL-27, $n=97$ & 2.82 & 0.95 & 8.40 & 0.062 & 3.02 & 0.94 & 9.64 & 0.063 \\
\hline \multicolumn{9}{|c|}{ Overall survival of all included patients } \\
\hline IL-6, $n=118$ & 2.81 & 1.46 & 5.40 & 0.002 & 2.99 & 1.54 & 5.81 & 0.001 \\
\hline IL-27, $n=97$ & 2.05 & 0.94 & 4.50 & 0.072 & 1.98 & 0.86 & 4.57 & 0.108 \\
\hline
\end{tabular}

$H R$ hazard ratio, $C I$ confidence interval
(Fig. 3e). The same was the case with the soluble sIL-6R $\alpha$ levels $(p=0.08)$ (Fig. 3f).

\section{IL-6 family cytokines and soluble receptors vs. overall survival (OS) with all patients included}

In Kaplan-Meier analysis, IL-6 values predicted OS $(p=0.001)$ (Fig. 4a). In a Cox multivariate survival analysis, including the gender, age and tumor size of the patient, a significant survival prediction was still determined (HR 2.99; CI 1.5-5.81; $p=0.002$ ) (Table 1). IL-27 showed no survival prediction with a Kaplan-Meier approach ( $p=0.066$ ) (Fig. 4b). Regarding Cox multivariate regression analysis (HR 1.98; CI 0.86-4.57; $p=0.11$ ) (Table 1), OS was not predicted. The model was tested and was stable for HR with regard to the IL-6 and IL-27 groups. 
Table 2 Outcome predictions from combined IL-6 and family cytokine members in Cox regression analyses

\begin{tabular}{|c|c|c|c|c|c|c|c|c|}
\hline & \multicolumn{4}{|c|}{$\begin{array}{l}\text { Multivariate (only cytokine/receptor } \\
\text { combined) }\end{array}$} & \multicolumn{4}{|c|}{$\begin{array}{l}\text { Multivariate including age, gender and } \\
\text { tumor size }\end{array}$} \\
\hline & \multirow[t]{2}{*}{ HR } & \multicolumn{2}{|c|}{$95 \% \mathrm{CI}$} & \multirow[t]{2}{*}{$p$ value } & \multirow[t]{2}{*}{ HR } & \multicolumn{2}{|l|}{$95 \% \mathrm{CI}$} & \multirow[t]{2}{*}{$p$ value } \\
\hline & & Lower & Upper & & & Lower & Upper & \\
\hline \multicolumn{9}{|c|}{ Recurrence in radically treated patients $(n=91)$} \\
\hline IL-6 & 6.64 & 1.80 & 24.4 & 0.004 & 25.5 & 3.04 & 213.4 & 0.003 \\
\hline IL-27 & 3.10 & 0.87 & 11.1 & 0.082 & 1.54 & 0.20 & 11.7 & 0.675 \\
\hline \multicolumn{9}{|c|}{ Disease-specific survival $(n=97)$} \\
\hline IL-6 & 7.47 & 2.26 & 24.7 & 0.001 & 10.8 & 2.62 & 44.4 & 0.003 \\
\hline IL-27 & 1.98 & 0.65 & 6.00 & 0.227 & 0.85 & 0.22 & 3.30 & 0.813 \\
\hline IL-6 & 10.1 & 3.07 & 33.4 & $<0.001$ & 20.7 & 5.25 & 81.4 & $<0.001$ \\
\hline IL-6R $\alpha$ & 0.17 & 0.021 & 1.31 & 0.089 & 0.068 & 0.007 & 0.66 & 0.020 \\
\hline gp130 & 1.11 & 0.24 & 5.12 & 0.889 & 2.64 & 0.45 & 15.4 & 0.281 \\
\hline
\end{tabular}

$H R$ hazard ratio, $C I$ confidence interval
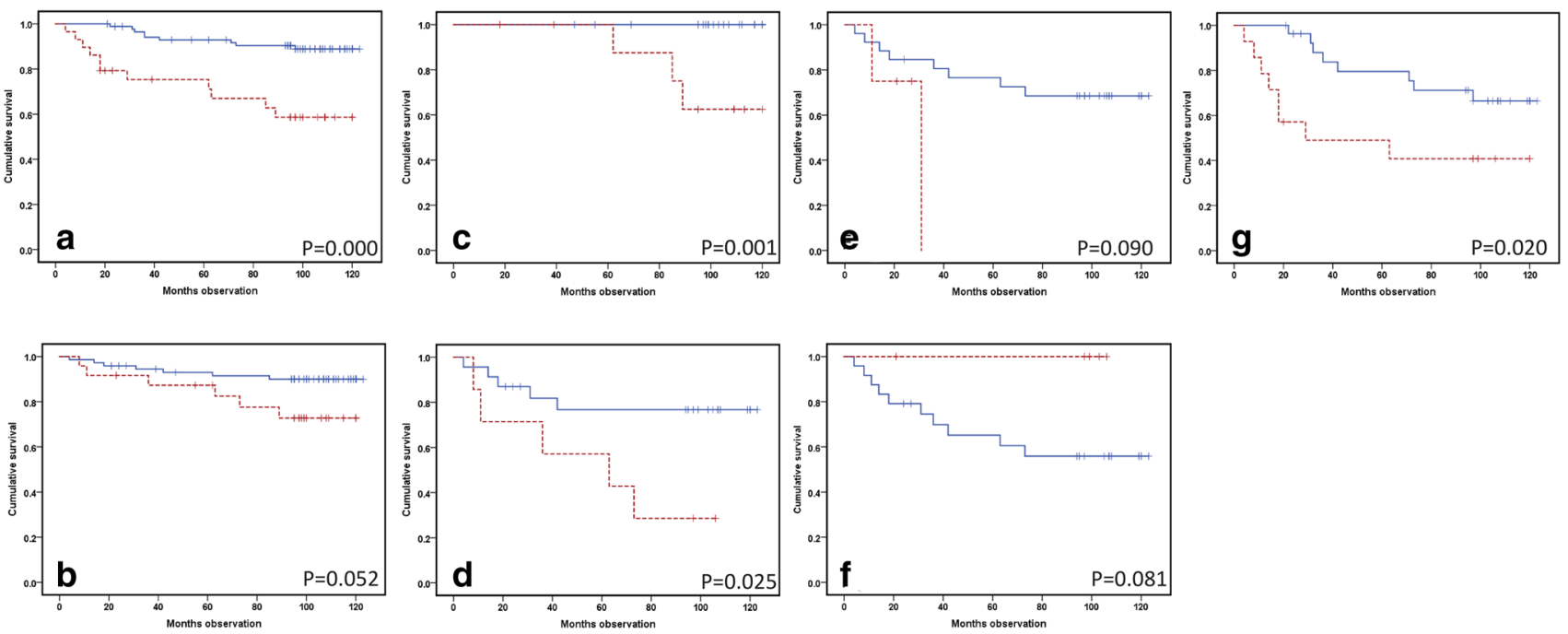

Fig. 3 Kaplan-Meier curves demonstrating disease-specific survival (DSS) prediction from IL-6, as well as related cytokines and receptors in pre-treatment RCC blood samples collected before surgical treatment. Analytes were measured simultaneously by Luminex technology. Low values are shown with a blue continuous line, and high values in red dotted lines. In addition, the graphs comprise $p$ values from log-rank tests. a IL-6 DSS prediction among 118 RCC patients (low $(<8 \mathrm{pg} / \mathrm{ml}): n=89$ and high $(\geq 8 \mathrm{pg} / \mathrm{ml})$ :

If the patients were grouped by tumor size, the IL-6 values in particular predicted survival among patients with medium-sized tumors (tumor diameter from 4 to $7 \mathrm{~cm})(p=0.018)$ (Fig. 4c), but not statistically significant $(p=0.063)$ among large tumors (Fig. 4e). If gp130 levels were studied in patients with large tumors only, a high gp130 level predicted a lower survival $(p=0.001)$ (Fig. $4 d)$. $n=29$ ). b IL-27 prediction of DSS in 97 RCC patients (low: $n=73$ and high: $n=24)$. c and $\mathbf{g}$ DSS prediction from IL-6 in mediumsized (4.1-7 cm, $n=37)$ and large ( $>7 \mathrm{~cm}, n=42)$ RCC tumors. The highest quartile is denoted by high $(n=10 / 14)$, and the remaining values low $(n=27 / 23)$. d-f IL-27, gp130 and IL6R alpha prediction of DSS in patients harboring a large $(>7 \mathrm{~cm}) \mathrm{RCC}$ tumor $(n=30)$. Quartiled analytes as above (high: $n=7 / 4 / 6$ and low: $n=23 / 26 / 24$ )

\section{Outcome dependent on IL-6 levels at the individual patient level}

We detected IL- $6>8 \mathrm{pg} / \mathrm{ml}$ in 29 patients: six of those with metastasis at the time of diagnosis, with seven of the remaining 23 patients presumed radically treated having had a subsequent RCC recurrence. Of those patients with a low IL-6 who died, or developed recurrent RCC disease $(n=10)$, only one had a RCC tumor $<7 \mathrm{~cm}$ at diagnosis. Five of the 10 patients with a high IL-6 who were still alive and without disease recurrence at the study closure, 

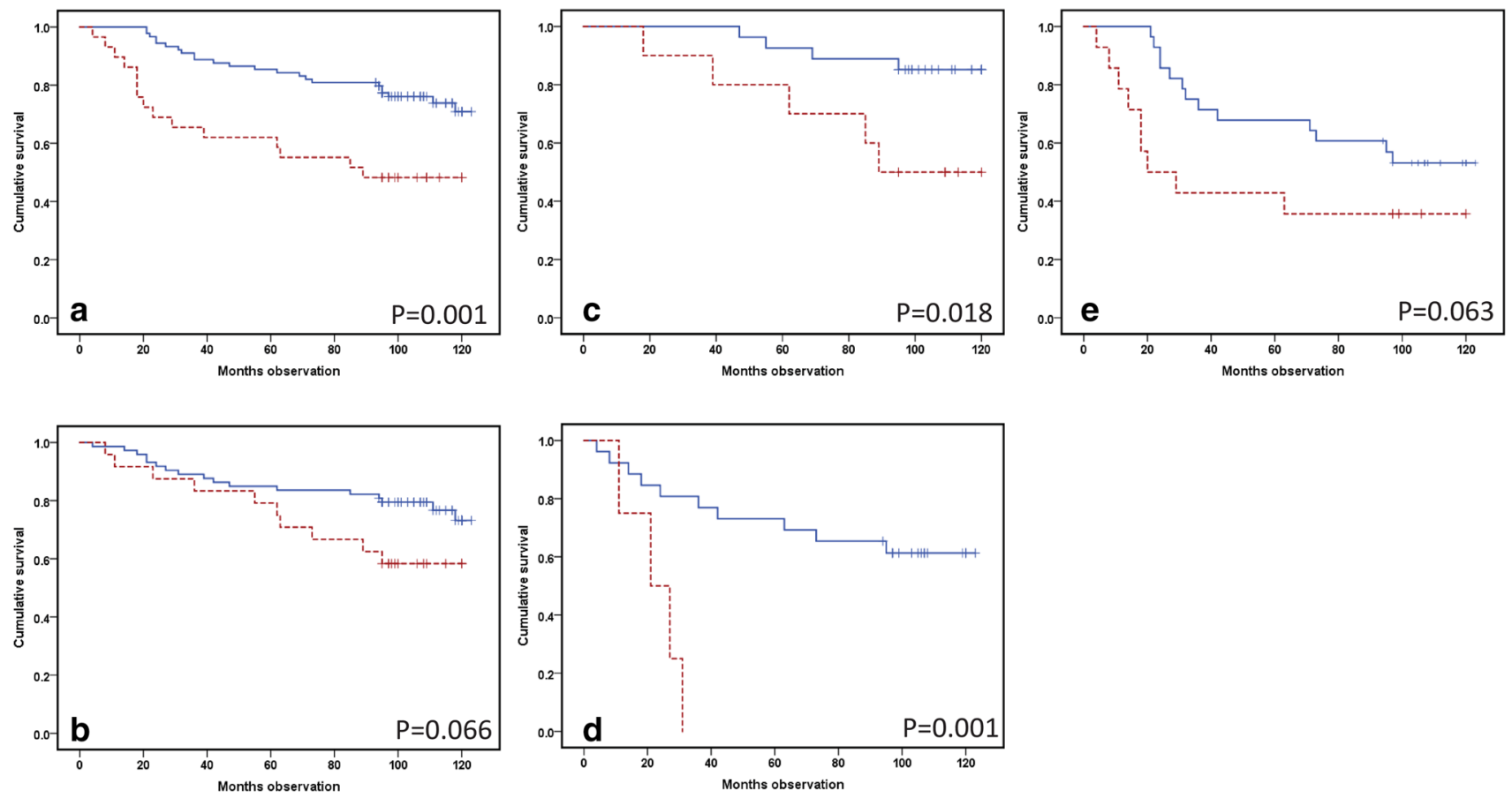

Fig. 4 Overall survival (OS) Kaplan-Meier curve predictions from IL- 6 and the family molecules IL-27 and gp130 by Luminex in serum collected before the surgical treatment of RCC patients. The blue continuous line visualizes low values (lower quartiles), with the high values in the red dotted line. Log-rank test $p$ values are included in the separate windows. a IL- 6 OS prediction among 118 RCC patients (low $(<8 \mathrm{pg} / \mathrm{ml}): n=89$ and high $(\geq 8 \mathrm{pg} / \mathrm{ml}): n=29)$. b IL-27 prediction of OS in 97 RCC patients (low: $n=73$ and high: $n=24$ ). c OS prediction from IL-6 in medium-sized [(4.1-7 cm, $n=37)$, $n=42]$ RCCs. Twenty-seven patients were designated as low, with 10 patients having a value within the highest quartile. $\mathbf{d}$ Prediction of OS from quartiled gp130 in RCC patients with a tumor size exceeding $7 \mathrm{~cm}$ (low: $n=26$ and high: $n=4$ ). e IL-6 OS prediction in large $(>7 \mathrm{~cm}, n=42)$ RCC tumors; 28 high value patients and 14 with a low value

Table 3 Description of immunohistochemical analyses, staining assessment and numbers

\begin{tabular}{|c|c|c|c|c|c|c|c|c|}
\hline & $-=0.0$ & $\pm=0.25$ & $\pm=0.5$ & $+=1.0$ & $+(+)=1.5$ & $++=2.0$ & $++(+)=2.5$ & $+++=3.0$ \\
\hline CD3-positive tumor lymphocytes & 1 & 0 & 5 & 11 & 4 & 2 & 4 & 1 \\
\hline CD3-positive lymphocytes in interphase zone & 3 & 0 & 7 & 10 & 2 & 4 & 1 & 1 \\
\hline CD68-positive cells in tumor & 0 & 0 & 3 & 7 & 7 & 6 & 5 & 0 \\
\hline CD68-positive interphase zone cells & 14 & 1 & 5 & 5 & 1 & 2 & 0 & 0 \\
\hline FoxP3 in tumor lymphocytes ${ }^{\mathrm{a}}$ & 9 & 14 & 2 & 2 & 0 & 0 & 0 & 0 \\
\hline FoxP3 in interphase zone lymphocytes ${ }^{a}$ & 9 & 8 & 6 & 3 & 0 & 1 & 0 & 0 \\
\hline FoxP3 in tumor cells ${ }^{\mathrm{a}}$ & 25 & 2 & 0 & 0 & 0 & 0 & 0 & 0 \\
\hline IL6 in tumor lymphocytes & 18 & 2 & 4 & 4 & 0 & 0 & 0 & 0 \\
\hline IL6 in interphase zone lymphocytes & 16 & 10 & 2 & 0 & 0 & 0 & 0 & 0 \\
\hline IL6 in tumor cells & 5 & 8 & 6 & 4 & 2 & 3 & 0 & 0 \\
\hline IL6 in vasculature & 1 & 2 & 1 & 3 & 2 & 6 & 1 & 12 \\
\hline IL6 receptor in tumor lymphocytes & 5 & 13 & 6 & 1 & 0 & 3 & 0 & 0 \\
\hline IL6R in interphase zone lymphocytes & 3 & 12 & 6 & 5 & 0 & 2 & 0 & 0 \\
\hline IL6R in tumor cells & 1 & 10 & 6 & 7 & 4 & 0 & 0 & 0 \\
\hline
\end{tabular}

Patient samples $(n=28)$ were scored in a semi-quantitative fashion, reviewed by an expert in pathology (LB) and further transformed into numeric values for statistical analyses according to the following: $+++=3,++(+)=2.5,++=2,+(+)=1.5,+=1, \pm=0.5,=0.25$, and $-=0.0$

${ }^{\mathrm{a}} n=27$ 
had either a second primary cancer or an autoimmune disease at diagnosis.

\section{Outcome by ROC analyses}

Both tumor diameter and IL-6 values predicted DSS and recurrence. According to IL-6 for recurrence, estimated areas under the curve (AUC) were $0.723 \pm 0.075(p=0.007)$ and $0.692 \pm 0.074(p=0.020)$, employing presumed radically treated or all patients, respectively. Regarding IL-27, the corresponding AUC results were $0.762 \pm 0.080(p=0.007)$ and $0.757 \pm 0.079(p=0.008)$, respectively (Fig. 5). Including only presumed radically treated patients and with large tumors (diameter $>7 \mathrm{~cm}$ ), the AUC were $0.908 \pm 0.069$ $(p=0.001)$ in the case of IL-27, and $0.707 \pm 0.098$ $(p=0.048)$ in the case of IL-6 (Fig. 5).

\section{IL-6 and IL-27 prediction of recurrence adjusted by Leibovich scores}

IL-6 and IL-27 levels were studied by Cox regression adjusted by Leibovich scores. The results showed that both
IL-6 $(p=0.01)$ and IL-27 $(p=0.014)$ still predicted survival following such an adjustment (Supplementary Table 3).

\section{Patients with high serum IL-6: outcome compared to tumor and tumor border (interphase) tissue CD3, CD68, IL-6 and IL6R-positive cells determined by immunohistochemistry}

By immunohistochemistry, we have determined the level of CD3, FoxP3, CD68, IL-6 and IL6R-positive cells in tumors from patients with high IL-6 serum values (Table 3 ). The following number of patients with at least a $10 \%(1+)$ expression on markers denoting cell characteristics were found at least at $1+$ levels: intra-tumor $\mathrm{CD} 3+$ lymphocytes: 22/28, interphase zone CD3 + lymphocytes: $18 / 28$, intra-tumor CD68+cells: 25/28, interphase zone CD68 + cells: $8 / 28$, FoxP3 + intra-tumor lymphocytes: 2/27, FoxP3 + interphase zone lymphocytes: 4/27 and FoxP3 + tumor cells: 0/27.

Regarding the present IL- 6 content of the various tumorassociated cells, the following were determined: intra-tumor lymphocytes: $4 / 28$, interphase zone lymphocytes: $0 / 28$, tumor cells $9 / 28$ and most density was seen in vascular cells:
Fig. 5 ROC recurrence.

Receiver operating characteristic (ROC) curves comparing recurrence prediction of IL-6, IL-27, and CRP in presumed radically treated (upper panel, $n=89$ ) and all (lower panel, $n=95$ ) RCC patients with such values, as analyzed in their serum ahead of surgical treatment. In both cases, 10 were regarded as positive. The right column shows results in patients with a tumor above $7 \mathrm{~cm}$, of which nine had a positive annotation. The blue continuous line represents IL-6, the red dotted line is IL-27, and green semihatched line indicated by CRP
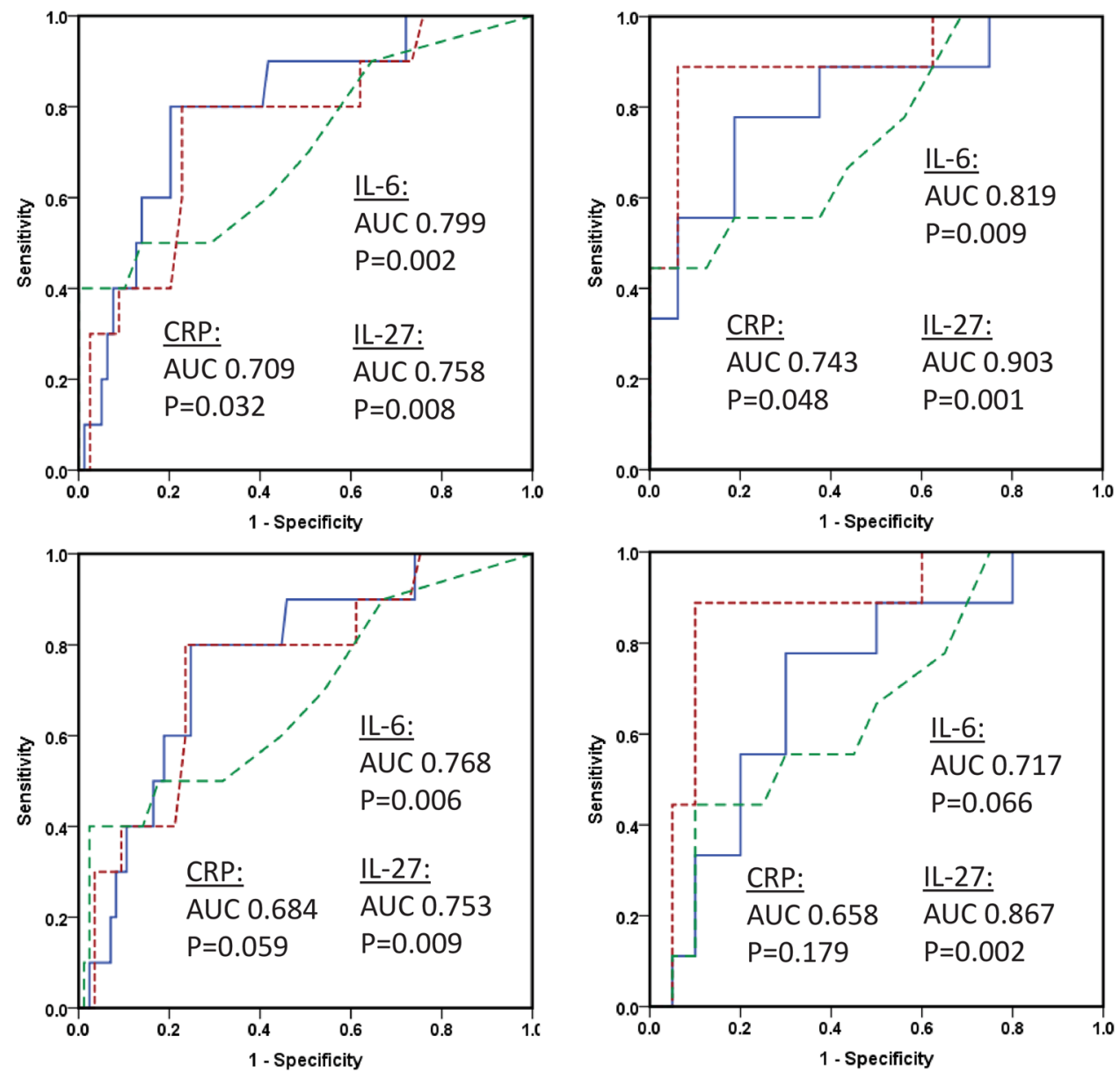
IL6 24/28. In the case of the IL6R, the following numbers were denoted: intra-tumor lymphocytes $4 / 28$, interphase zone lymphocytes: 7/28 and tumor cells: 11/28.

The cellular derived measurement did not substantially correlate to tumor diameter or CRP levels. The various above-mentioned variables were also tested regarding prognostic value. In particular, the extent of T lymphocytes (CD3 + cells) infiltration in the tumors predicted survival. A high CD3 + value predicted a decreased survival. This was valid concerning recurrence $(p=0.017)$ and DSS $(p=0.032)$, but not for OS (Fig. 6).

\section{Discussion}

High IL-6 and IL-27 serum levels predicted a worse prognosis. Among clinically presumed radically treated patients, a high IL-6 and high IL-27 strongly predicted a recurrence in both univariate and multivariate analyses. IL-6 also predicted DSS and OS. Overall, the predictions among patients with large tumors (diameter $>7 \mathrm{~cm}$ ) were excellent regarding both high IL-6 and IL-27 values. Of the soluble receptors studied, high gp130 serum levels predicted a worse OS among the RCC patients with large tumors.

The cytokine levels best predicted survival among patients with large tumors. These findings support that a high cytokine value points to a biologically aggressive tumor, more than a low differentiated tumor $[8,10,24]$.

Patients with high levels of IL-6, but only with a clinically localized disease, were more likely to die from their RCC, and also had an increased risk of dying of any cause compared to those with low IL-6 serum values. Our findings are in agreement with those of Ljungberg et al. [25], Hrab et al. [26] and Blay et al. [27].

Increased IL-27 levels predicted recurrence and DSS, especially among patients with a tumor diameter $>7 \mathrm{~cm}$.
To the best of our knowledge, this has not been shown in other published studies. Only one study has thus far been published on IL-27 and RCC, which showed that patients with specific polymorphisms of IL-27 are more susceptible to RCC [17].

Soluble IL-6R $\alpha$ may bind to IL-6, and secondarily bind to gp130 receptors on the surface of cells, in this way stimulating pro-inflammatory functions through transactivation. On the other hand, cis-activation is mostly immune inhibitory [28]. We have not shown negative prognostic effects of increased serum IL-6R $\alpha$ among RCC patients, supporting that IL-6 promotes inflammation in RCC tumors as a mechanism of IL-6-driven carcinogenesis. Soluble gp130 binds the soluble IL-6/IL-6R $\alpha$ complex [29], and presumably acts as an IL-6/IL-6R $\alpha$ decoy receptor [28]. We have demonstrated a negative prognostic value of increased gp130 in the serum of patients with larger tumors, which is the opposite of what should be expected. However, gp130 is present on most cells [28], and the increased serum soluble gp130 may be caused by generally increased tumor cellular turnover, which then basically drives the worse prognosis.

The combined effect of soluble IL-6R $\alpha$, gp130 and IL-6 as to prognosis seems to be complex. Regarding small tumors, the results are as expected, but concerning larger tumors s-gp130 levels paint another picture more consistent with that reflected by s-gp130 levels, e.g., cellular proliferation. Furthermore, the similar survival prediction of IL-6 and IL-27 suggests that this association is limited to cells actually carrying the IL-6 receptor on the surface, as no soluble IL-27 receptor has so far been recognized.

IL-6 and the IL6R may also be determined in tumor tissue [20]. Fu et al. [20] have shown that the expression on tumor cells of IL-6/IL6R worsens the prognosis. We have verified that both the IL-6 and IL6R may be found on cancer cells from RCC patients with high IL-6. Hence, it is supported that IL-6 may act directly on the tumor with a subsequent
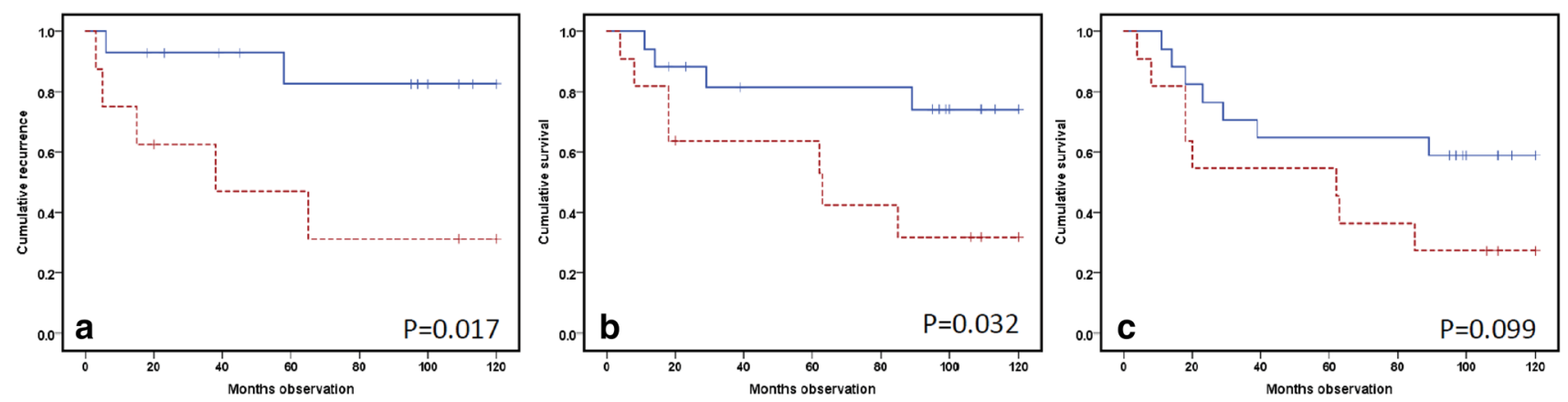

Fig. 6 Outcome predictions: a recurrence, b disease-specific survival, and $\mathbf{c}$ overall survival of total 28 RCC patients from immunohistochemical quantification of tumor CD3-positive $\mathrm{T}$ lymphocytes in surgical resection specimen. The expression levels were qualitatively scored by an experienced pathologist and further dichotomized by median value into high (All patients: $n=11 /$ radically treated: $n=8)$ and low $(n=17$ for all patients $n=14$ for radically treated). $p$ values come from log-rank tests 
worse RCC prognosis, both in an autocrine and paracrine manner [30].

We have also shown that among patients with a high IL-6, a surprisingly high expression of IL-6 was found in vascular cells, i.e., endothelial and smooth muscle cells, thereby suggesting that these cells produce IL-6. Endothelial cells are presumably stimulated by VEGF from the tumor [31], with this representing a possible loop where the tumor may become autocrine stimulated.

High IL-6 values in serum also signal a worse OS, and as such, IL-6 values are coupled with many serious diseases [32]. IL-6 is elevated in hypertension, as well as being associated with a higher incidence of future cardiovascular events and mortality [33]. This may partly explain the shown overall survival prediction.

Moreover, we have studied levels of T lymphocyte tumor infiltration and presence in the tumor periphery in a subgroup of patients selected by high IL-6 serum levels. A high $\mathrm{T}$ lymphocyte count predicted an increased recurrence and decreased survival. Nevertheless, T regulatory lymphocytes, i.e., Fox P3 lymphocytes, were not to any extent found within the tumor. This is in line with what has previously been shown in head and neck squamous cell carcinomas [34], namely that survival prediction in solid tumors is likely dependent on several immune-related dimensions, like presently one associated with general inflammation through IL-6, and another associated with specific immunity though $\mathrm{T}$ lymphocytes [35].

RCC survival prediction is expected to be secondary to factors like the ones included in the Leibovich scores. Leibovich is a composite score, including tumor size, pathological $\mathrm{T}$ and $\mathrm{N}$ stage, Fuhrman nuclear grading and histological necrosis [36]. With the IL-6 and IL-27 recurrence prediction adjusted by the Leibovich score, both of these cytokine levels still predicted survival.

Clinically, the present results may be relevant. When applying $8 \mathrm{pg} / \mathrm{ml}$ IL-6 levels as a cut-off between high and low IL-6 values, 29 patients had high IL-6 values. Six out of nine patients with detectable metastasis at diagnosis had high IL-6 values, as had seven of 14 individuals who subsequently developed RCC metastases. Several other patients presumably had other specific causes of their increased IL-6. Of those patients with a low IL-6 who died, or developed recurrent RCC disease $(n=10)$ only one had a RCC tumor with a diameter $<7 \mathrm{~cm}$ at diagnosis. The IL-6 values may therefore be utilized at the individual level to sort patients with both a high and low risk of dying because of RCC disease.

Furthermore, ROC analyses suggested that a high IL-27 and IL- 6 score predicted a recurrence with both a high sensitivity and specificity, especially as measured in patients with larger tumors. Thus, we have demonstrated that IL-6 and IL-27 may be utilized as biomarkers to identify both a high- and low-risk recurrence of RCC patients at the time of diagnosis.

Patients with high IL-6/IL-27 values at diagnosis may be good candidates for adjuvant treatment with, e.g., VEGF inhibitors [37], as well as with anti-IL-6 therapy such as Siltuximab [38]. The agent Siltuximab ( $\alpha$ IL-6) has shown promising results in phase I/II studies for metastatic RCC [38]. It is even possible that a combined blockage of IL-6/ IL-27/VEGF would have achieved better results. The results of our study also demonstrate the need for future clinical studies of therapies investigating blockage of gp130 pathways, i.e., bazedoxifene, which blocks p-STAT3 inhibitor [39], and also combined with other blockers like VEGF-TKIs [40] to prolong survival in patients with RCC [41]. However, it should be borne in mind that babies born with a defect gp130 receptor may suffer from extended Stüve-Wiedemann syndrome, which is a serious, often lethal syndrome [42]. Thus, to block gp130 may have serious side effects, making such treatment impossible. Our results also add to knowledge inspiring $\mathrm{T}$ cell boosting therapy to be further developed. In any case, the role of IL-27 biology in RCC should be studied judged against the background that new templates for biological therapy in RCC therapy are urgently needed [43].

This study includes a limited number of patients. Therefore, the analyses, especially on the sub-group level showing negative results, must be interpreted with caution. We have measured the cytokines and soluble receptor levels just once. In particular, cytokines in the blood may have a short half-life [44], as a broader picture could have been painted with additional measuring points.

\section{Conclusions}

IL-6 and IL-27 have been shown to have a role in RCC biology through the predictive ability of recurrence and disease-specific survival in otherwise radically treated RCC patients. We believe that patients with a high IL-6 and IL-27 will be good candidates on which to base a biological therapy of RCC. Finally, both these cytokines hold promise for being important in relation to risk stratification regarding RCC prognosis, and thereby a need for treatment.

Acknowledgements Open Access funding provided by University of Bergen.

Author contributions GG: Project development, data collection and analysis and writing the manuscript; HA: Analysis and figures, manuscript editing; LB: Data collection and manuscript editing; KMH: Manuscript editing; HJA: Project development, analysis and manuscript editing; ØB: analysis and manuscript editing; THAT: analysis and data editing; CB: Project development, data collection and 
analysis and manuscript editing. All authors have read and approved the final manuscript.

Funding The study has been carried out with funding from the institutions mentioned on the title page.

Availability of data and material The approval from the ethical committee and informed consent do not cover a full open publication of the dataset. The raw data will be made available in unidentified form on request, and if needed contact the corresponding author.

\section{Compliance with ethical standards}

Conflict of interest The authors declare that they have no competing interests. The authors alone are responsible for the content and writing of the paper.

Ethics approval The study was approved by the Regional Committee for Medical Research Ethics in Western Norway (78/05), and the database was approved by the Norwegian Social Science Data Services.

Consent to participate All patients were given oral and written information about the study, and they gave written informed consent.

Open Access This article is licensed under a Creative Commons Attribution 4.0 International License, which permits use, sharing, adaptation, distribution and reproduction in any medium or format, as long as you give appropriate credit to the original author(s) and the source, provide a link to the Creative Commons licence, and indicate if changes were made. The images or other third party material in this article are included in the article's Creative Commons licence, unless indicated otherwise in a credit line to the material. If material is not included in the article's Creative Commons licence and your intended use is not permitted by statutory regulation or exceeds the permitted use, you will need to obtain permission directly from the copyright holder. To view a copy of this licence, visit http://creativecommons .org/licenses/by/4.0/.

\section{References}

1. Allemani C et al (2018) Global surveillance of trends in cancer survival 2000-2014 (CONCORD-3): analysis of individual records for 37513025 patients diagnosed with one of 18 cancers from 322 population-based registries in 71 countries. Lancet 391(10125):1023-1075

2. Bray F et al (2018) Global cancer statistics 2018: GLOBOCAN estimates of incidence and mortality worldwide for 36 cancers in 185 countries. CA Cancer J Clin 68(6):394-424

3. Heidegger I, Pircher A, Pichler R (2019) Targeting the tumor microenvironment in renal cell cancer biology and therapy. Front Oncol 9:490-490

4. Tannir NM, Pal SK, Atkins MB (2018) Second-line treatment landscape for renal cell carcinoma: a comprehensive review. Oncologist 23(5):540-555

5. Mazza C, Escudier B, Albiges L (2017) Nivolumab in renal cell carcinoma: latest evidence and clinical potential. Ther Adv Med Oncol 9(3):171-181

6. Mihara $M$ et al (2012) IL-6/IL-6 receptor system and its role in physiological and pathological conditions. Clin Sci 122(4):143-159
7. Lippitz BE, Harris RA (2016) Cytokine patterns in cancer patients: a review of the correlation between interleukin 6 and prognosis. Oncoimmunology 5(5):e1093722

8. Scheller J, Garbers C, Rose-John S (2014) Interleukin-6: from basic biology to selective blockade of pro-inflammatory activities. Semin Immunol 26(1):2-12

9. Tvedt THA et al (2017) Interleukin-6 in allogeneic stem cell transplantation: its possible importance for immunoregulation and as a therapeutic target. Front Immunol 8:667

10. Taniguchi K, Karin M (2014) IL-6 and related cytokines as the critical lynchpins between inflammation and cancer. Semin Immunol 26(1):54-74

11. Lamertz L et al (2018) Soluble gp130 prevents interleukin-6 and interleukin-11 cluster signaling but not intracellular autocrine responses. Sci Signal 11(550):eaar7388

12. Rose-John S (2018) Interleukin-6 family cytokines. Cold Spring Harb Perspect Biol 10(2):a028415

13. Jones SA, Jenkins BJ (2018) Recent insights into targeting the IL-6 cytokine family in inflammatory diseases and cancer. Nat Rev Immunol 18(12):773-789

14. Polimeno $\mathrm{M}$ et al (2013) Regulatory $\mathrm{T}$ cells, interleukin (IL)6, IL-8, vascular endothelial growth factor (VEGF), CXCL10, CXCL11, epidermal growth factor (EGF) and hepatocyte growth factor (HGF) as surrogate markers of host immunity in patients with renal cell carcinoma. BJU Int 112(5):686-696

15. Dosquet $C$ et al (1997) Are angiogenic factors, cytokines, and soluble adhesion molecules prognostic factors in patients with renal cell carcinoma? Clin Cancer Res 3(12):2451-2458

16. Johnson TV, Abbasi A, Owen-Smith A, Young AN, Kucuk O, Harris WB, Osunkoya AO, Ogan K, Pattaras J, Nieh PT, Marshall FF, Master VA (2010) Postoperative better than preoperative $\mathrm{C}$-reactive protein at predicting outcome after potentially curative nephrectomy for renal cell carcinoma. Urology 76:766

17. $\mathrm{Pu} \mathrm{Y}$ et al (2015) Association between polymorphisms in IL27 gene and renal cell carcinoma. Biomarkers 20(3):202-205

18. Fabbi M, Carbotti G, Ferrini S (2017) Dual roles of IL-27 in cancer biology and immunotherapy. Mediat Inflamm 2017:3958069-3958069

19. Parker WP et al (2017) Application of the stage, size, grade, and necrosis (SSIGN) score for clear cell renal cell carcinoma in contemporary patients. Eur Urol 71(4):665-673

20. Fu $\mathrm{Q}$ et al (2015) Prognostic value of interleukin-6 and interleukin-6 receptor in organ-confined clear-cell renal cell carcinoma: a 5-year conditional cancer-specific survival analysis. Br J Cancer 113(11):1581-1589

21. Beisland $C$ et al (2015) Contemporary external validation of the Leibovich model for prediction of progression after radical surgery for clear cell renal cell carcinoma. Scand J Urol 49(3):205-210

22. Beisland $\mathrm{C}$ et al (2016) A prospective risk-stratified follow-up programme for radically treated renal cell carcinoma patients: evaluation after 8 years of clinical use. World J Urol 34(8): 1087-1099

23. Fuhrman SA, Lasky L, Limas C (1982) Prognostic significance of morphologic parameters in renal cell carcinoma. Am J Pathol 6:655-663

24. Yoshida $N$ et al (2002) Interleukin-6, tumour necrosis factor $\alpha$ and interleukin- $1 \beta$ in patients with renal cell carcinoma. Br J Cancer 86(9):1396-1400

25. Ljungberg B, Grankvist K, Rasmuson T (1997) Serum interleukin-6 in relation to acute-phase reactants and survival in patients with renal cell carcinoma. Eur J Cancer 33(11):1794-1798

26. Hrab $M$ et al (2013) Interleukin-6 (IL-6) and C-reactive protein (CRP) concentration prior to total nephrectomy are prognostic factors in localized renal cell carcinoma (RCC). Rep Pract Oncol Radiother 18(5):304-309 
27. Blay J-Y et al (1992) Serum level of interleukin 6 as a prognosis factor in metastatic renal cell carcinoma. Cancer Res 52(12):3317-3322

28. Silver JS, Hunter CA (2010) gp130 at the nexus of inflammation, autoimmunity, and cancer. J Leukoc Biol 88(6):1145-1156

29. Murakami M, Kamimura D, Hirano T (2019) Pleiotropy and specificity: insights from the interleukin 6 family of cytokines. Immunity 50(4):812-831

30. Kumar A et al (2018) Renal cell carcinoma: molecular aspects. Indian J Clin Biochem IJCB 33(3):246-254

31. Ferrara N (2009) Vascular endothelial growth factor. Arterioscler Thromb Vasc Biol 29(6):789-791

32. Jiang $Y$ et al (2019) Inflammatory anemia-associated parameters are related to 28-day mortality in patients with sepsis admitted to the ICU: a preliminary observational study. Ann Intensive Care 9(1):67

33. Didion SP (2017) Cellular and oxidative mechanisms associated with interleukin-6 signaling in the vasculature. Int J Mol Sci 18(12): 2563

34. Aarstad HJ et al (2017) In vitro monocyte IL-6 secretion levels following stimulation with autologous spheroids derived from tumour or benign mucosa predict long-term survival in head and neck squamous cell carcinoma patients. Scand J Immunol 85(3):211-219

35. Stenzel PJ et al (2019) Prognostic and predictive value of tumorinfiltrating leukocytes and of immune checkpoint molecules PD1 and PDL1 in clear cell renal cell carcinoma. Transl Oncol 13(2):336-345

36. Leibovich BC et al (2018) Predicting oncologic outcomes in renal cell carcinoma after surgery. Eur Urol 73(5):772-780
37. Yang JC et al (2003) A randomized trial of bevacizumab, an antivascular endothelial growth factor antibody, for metastatic renal cancer. N Engl J Med 349(5):427-434

38. Rossi JF et al (2010) A phase I/II study of siltuximab (CNTO 328), an anti-interleukin-6 monoclonal antibody, in metastatic renal cell cancer. Br J Cancer 103(8):1154-1162

39. Wei J et al (2019) Bazedoxifene as a novel GP130 inhibitor for colon cancer therapy. J Exp Clin Cancer Res 38(1):63

40. Gill DM et al (2018) Overview of current and future first-line systemic therapy for metastatic clear cell renal cell carcinoma. Curr Treat Options Oncol 19(1):6

41. Ishibashi $\mathrm{K}$ et al (2018) Interleukin-6 induces drug resistance in renal cell carcinoma. Fukushima J Med Sci 64(3):103-110

42. Chen Y-H et al (2020) Absence of GP130 cytokine receptor signaling causes extended Stüve-Wiedemann syndrome. J Exp Med 217(3):e20191306

43. Lenis AT et al (2018) Adjuvant therapy for high risk localized kidney cancer: emerging evidence and future clinical trials. J Urol 199(1):43-52

44. Aziz N et al (2016) Stability of cytokines, chemokines and soluble activation markers in unprocessed blood stored under different conditions. Cytokine 84:17-24

Publisher's Note Springer Nature remains neutral with regard to jurisdictional claims in published maps and institutional affiliations. 